\title{
Constraints on the density dependence of the symmetry energy from heavy ion collisions
}

\author{
M.B. Tsang, ${ }^{1}$ Z. Chajecki, ${ }^{1}$ D. Coupland,${ }^{1}$ P. Danielewicz,${ }^{1}$ F. Famiano, ${ }^{2}$ R. Hodges,${ }^{1}$ \\ M. Kilburn, ${ }^{1}$ F. Lu, ${ }^{3}$ W.G. Lynch, ${ }^{1}$ J. Winkelbauer, ${ }^{1}$ M. Youngs,${ }^{1}$ Y.X. Zhang ${ }^{4}$ \\ ${ }^{1}$ National Superconducting Cyclotron Laboratory \\ Michigan State University, East Lansing, MI 48824, USA \\ ${ }^{2}$ Physics Dept, Western Michigan University, Kalamazoo, MI USA \\ ${ }^{3}$ School of Physics, Peking University, Beijing 100871, China \\ ${ }^{4}$ China Institute of Atomic Energy, Beijing 102413, China
}

March 22, 2022

\begin{abstract}
Constraints on the Equation of State for symmetric matter (equal neutron and proton numbers) have been extracted from energetic collisions of heavy ions over a range of energies. Collisions of neutron-deficient and neutron-rich heavy ions now provide initial constraints on the EoS of neutron-rich matter at sub-saturation densities from isospin diffusions and neutron proton ratios. This article reviews the experimental constraints on the density dependence of Symmetry Energy at sub-saturation density.
\end{abstract}

\section{Introduction}

The Equation of State (EoS) of cold nuclear matter can be written as the sum of the energy per nucleon of symmetric matter and a symmetry energy term, $E_{\delta}$

$$
E(\rho, \delta)=E_{0}(\rho, \delta=0)+E_{\delta}
$$

where $\delta=\left(\rho_{n}-\rho_{p}\right) / \rho$ is the asymmetry; $\rho_{n}, \rho_{p}$ and $\rho$ are the neutron, proton and nucleon densities, respectively. The first term on the RHS, $E_{0}(\rho, \delta=0)$, is the EoS term for symmetric nuclear matter with equal fractions of neutrons and protons. Significant constraints on the symmetric matter EoS at $1 \leq \rho / \rho_{0} \leq 4.5$ have been obtained from measurements of collective flow [1] and Kaon production [2]. However, well-determined constraints on the symmetry term, $E_{\delta}=S\left(\rho, \delta^{2}\right)$, are few. $S(\rho)$ describes the density dependence of $E_{\delta}$.

Since large variations in nuclear density can be attained momentarily in nuclear collisions, constraints on the Equation of State (EoS) can be obtained by comparing measurements to transport calculations of such collisions. The symmetry energy has been recently probed at sub-saturation densities via isospin diffusion [3, 4], and by double ratios involving neutron and proton energy spectra [5]. These two observables largely reflect the transport of nucleons under the combined influence of the mean fields and 
the collisions induced by residual interactions; thus, they should be within the predictive capabilities of transport theory.

This article focuses on investigations of the symmetry energy at sub-saturation density. The constraints obtained in heavy ion collisions are compared to constraints obtained from nuclear structure studies, from Isobaric Analog States, Sn isotope skins, Pygmy Dipole Resonance experiments, and the Giant Dipole Resonance data. I will briefly discuss investigations of the density dependence of symmetry energy at supranormal density.

\section{ImQMD: Transport calculations for heavy ion collisions}

Transport models have been used to describe the dynamics of heavy ion collisions. We chose to use the ImQMD code mainly because of its ability to describe fragments which are quite important in describing isospin observables. In the QMD model, nucleons are represented by Gaussian wavepackets. The mean fields acting on these wavepackets are derived from an energy functional with the potential energy U that includes the full Skyrme potential energy with only the spin-orbit term omitted:

$$
U=U_{\rho}+U_{m d}+U_{\text {coul }} ; U_{\rho, m d}=\int u_{\rho, m d} d^{3} r
$$

where, $U_{\text {coul }}$ is the Coulomb energy and $U_{\rho, m d}$ represents the nuclear contributions in local form and

$$
u_{\rho}=\frac{\alpha}{2} \frac{\rho^{2}}{\rho_{0}}+\frac{\beta}{\eta+1} \frac{\rho^{\eta+1}}{\rho_{0}^{\eta}}+\frac{g_{\text {sur }}}{2 \rho_{0}}(\nabla \rho)^{2}+\frac{g_{\text {sur }, i s o}}{\rho_{0}}\left(\nabla\left(\rho_{n}-\rho_{p}\right)\right)^{2}+\frac{C_{s}}{2}\left(\frac{\rho}{\rho_{0}}\right)^{\gamma_{i}} \delta^{2} \rho+g_{\rho \tau} \frac{\rho^{8 / 3}}{\rho_{0}^{5 / 3}}
$$

where the asymmetry $\delta$, and $\rho_{n}$ and $\rho_{p}$ are the neutron and proton densities, respectively. A symmetry kinetic energy density of the form $\frac{C_{s, k}}{2}\left(\frac{\rho}{\rho_{0}}\right)^{2 / 3} \delta^{2} \rho$ and symmetry potential energy density of the form $\frac{C_{s, p}}{2}\left(\frac{\rho}{\rho_{0}}\right)^{\gamma} \delta^{2} \rho$ were used in the following transport model comparisons. The energy density associated with the mean-field momentum dependence is represented by

$$
u_{m d}=\frac{1}{2 \rho_{0}} \sum_{N_{1}, N_{2}} \frac{1}{16 \pi^{6}} \int d^{3} p_{1} d^{3} p_{2} f_{N_{1}}\left(\vec{p}_{1}\right) f_{N_{2}}\left(\vec{p}_{2}\right) 1.57\left[\ln \left(1+5 \times 10^{-4}(\Delta p)^{2}\right)\right]^{2}
$$

where $f_{N}$ are nucleon Wigner functions, $\Delta p=\left|\overrightarrow{p_{1}}-\overrightarrow{p_{2}}\right|$, the energy is in MeV and momenta are in $\mathrm{MeV} / \mathrm{c}$. The resulting interaction between wavepackets is described in Ref. 6]. Unless otherwise noted, we use $\alpha=-356 \mathrm{MeV}, \beta=303 \mathrm{MeV}$ and $\eta=7 / 6$, corresponding to a isoscalar compressibility constant of $\mathrm{K}=200 \mathrm{MeV}$, and $g_{\text {sur }}=19.47 \mathrm{MeVfm}^{2}, g_{\text {suriso }}=-11.35 \mathrm{MeVfm}^{2}, C_{s, k}=24.9 \mathrm{MeV}, C_{s, p}=35.19 \mathrm{MeV}$, and $g_{\rho \tau}=0 \mathrm{MeV}$.

These calculations use isospin-dependent in-medium nucleon-nucleon scattering cross sections in the collision term and Pauli blocking effects that are described in [7, 8]. Cluster yields are calculated by means of the coalescence model widely used in QMD calculations in which particles with relative momenta smaller than $P_{0}$ and relative distances smaller than $R_{0}$ are coalesced into one cluster. In the present work, values of $R_{0}=3.5 \mathrm{fm}$ and $P_{0}=250 \mathrm{MeV} / \mathrm{c}$ are employed.

\section{Experimental constraints from heavy ion collisions}

We turn our attention first to the interpretation of neutron/proton double ratio data, which derives its sensitivity to the symmetry energy from the opposite sign of the symmetry force for neutrons as compared to protons [9]. First experimental comparisons of neutron and proton spectra in Ref. [5] used 
a double ratio in order to reduce sensitivity to uncertainties in the neutron detection efficiencies and to relative uncertainties in energy calibrations of neutrons and protons. This double ratio,

$$
D R(n / p)=R_{n / p}(A) / R_{n / p}(B)=\frac{d M_{n}(A) / d E_{c . m .}}{d M_{p}(A) / d E_{c . m .}} \cdot \frac{d M_{n}(B) / d E_{c . m .}}{d M_{p}(B) / d E_{c . m .}},
$$

was constructed by measuring the energy spectra, $d M / d E_{c . m}$., of neutrons and protons for two systems $\mathrm{A}$ and $\mathrm{B}$ characterized by different isospin asymmetries. The star symbols in the left panel of Figure 1 show the double ratios measured at $70^{\circ} \leq \theta_{C M} \leq 110^{\circ}$ as a function of nucleon center-of-mass (c.m.) energy for central collisions of ${ }^{124} S n+{ }^{124} S n$ and ${ }^{112} S n+{ }^{112} S n$ at E/A=50 MeV.

We have performed calculations at an impact parameter of $b=1,2$ and $3 \mathrm{fm}$ at an incident energy of $50 \mathrm{MeV}$ per nucleon for two systems: $\mathrm{A}={ }^{124} S n+{ }^{124} S n$ and $\mathrm{B}={ }^{112} S n+{ }^{112} S n$. About 60,000 events are simulated for each impact parameter. Within the statistical uncertainties, the double ratio observable, $\operatorname{DR}(\mathrm{n} / \mathrm{p})$, is nearly independent of the impact parameters within the range of $0 \leq \mathrm{b} \leq 5 \mathrm{fm}$. The lines in the left panel of Figure 1 show the predicted double ratios $\operatorname{DR}(\mathrm{np})=R_{n / p}(124) / R_{n / p}(112)$ as a function of the c.m. energy of nucleons emitted at $70^{\circ} \leq \theta_{C . M} \leq 110^{\circ}$ for $\gamma_{i}=0.35,0.5,0.75,1$ and 2 . The uncertainties for these calculations in Figure 1 are statistical. As fewer nucleons are emitted at high energy, the uncertainties increase with increasing energy. Despite the large experimental uncertainties, especially for the higher energy $(>40 \mathrm{MeV})$ data, the trends and magnitudes of the data points definitely rule out the very soft ( $\gamma_{i}=0.35$, dotted line with open diamond points) and very stiff ( $\gamma_{i}=2$, dotted line with closed diamond symbols) density-dependent symmetry terms. We find the $2 \sigma$ uncertainty range of values for $\gamma_{i}$ to be $0.4 \leq \gamma_{i} \leq 1.04$, corresponding to an increase in $\chi^{2}$ by 4 above its minimum of $\chi^{2} \sim 2.1$ near $\gamma_{i}=0.7$.

The density dependence of the symmetry energy can also be probed in peripheral collisions between two nuclei with different isospin asymmetries by examining the diffusion of neutrons and protons across the neck that joins the nuclei. This "isospin diffusion" generally continues until the two nuclei separate or until the chemical potentials for neutrons and protons in both nuclei become equal. To isolate diffusion effects from other effects such as pre-equilibrium emission, Coulomb effects, and secondary decays, measurements of isospin diffusion compare "mixed" collisions involving a neutron-rich nucleus A and a neutron-deficient nucleus B to the "symmetric" collisions involving A+A and B+B. The degree of isospin equilibration in such collisions can be quantified by rescaling the isospin observable $\mathrm{X}$ according to the isospin transport ratio $R_{i}(X)[3]$ given by

$$
R_{i}(X)=2 \frac{X-\left(X_{A+A}+X_{B+B}\right) / 2}{X_{A+A}-X_{B+B}}
$$

In the absence of isospin diffusion, $R_{i}\left(X_{A+B}\right)=R_{i}\left(X_{A+A}\right)=1$ for a collision involving a neutron-rich projectile on a proton-rich target. Likewise, without diffusion, $R_{i}\left(X_{B+B}\right)=R_{i}\left(X_{B+A}\right)=-1$. On the other hand, if isospin equilibrium is achieved for roughly equal sized projectiles and target nuclei, $R_{i}\left(X_{A+B}\right)=R_{i}\left(X_{B+A}\right) \approx 0$. Using ImQMD model, we investigate $R_{i}(\delta)$ as a function of the rapidity of the emitted particles. The results of the calculations are shown by the lines in the right panel of Figure 2. The data, represented by the star symbols, are obtained from the yield ratios of the mirror nuclei pair, ${ }^{7} \mathrm{Li}$ and ${ }^{7} \mathrm{Be}, R_{7}=R_{i}\left(X=\ln \left(Y\left({ }^{7} \mathrm{Li}\right) / Y\left({ }^{7} \mathrm{Be}\right)\right)\right)$ [4. This calculation reproduces the shapes and magnitudes of the rapidity dependence of the isospin transport ratios $R_{7}$. The $\chi^{2}$ analysis brackets $\gamma_{i}$ values to lie in the region $0.4 \leq \gamma_{i} \leq 1.0 \mathrm{using}$ the same $2 \sigma$ criterion. There is substantial overlap between the constraints obtained from isospin diffusion and double neutron and proton yield ratios.

Constraints on the exponent $\gamma_{i}$ depend on the symmetry energy at saturation density, $S_{0}=S\left(\rho_{0}\right)=$ $0.5 *\left(C_{s, k}+C_{s, k}\right)$. Increasing $S_{0}$ has the same effect on the isospin transport ratio as decreasing $\gamma_{i}$. Thus $S_{0}$ and $\gamma_{i}$ cannot be determined independently. To provide constraints on $S_{0}$ and $\gamma_{i}$ parameters, contours with constant $\chi^{2}$ values can be created by doing two-dimensional $\chi^{2}$ analysis in the $\gamma_{i}-S_{0}$ 


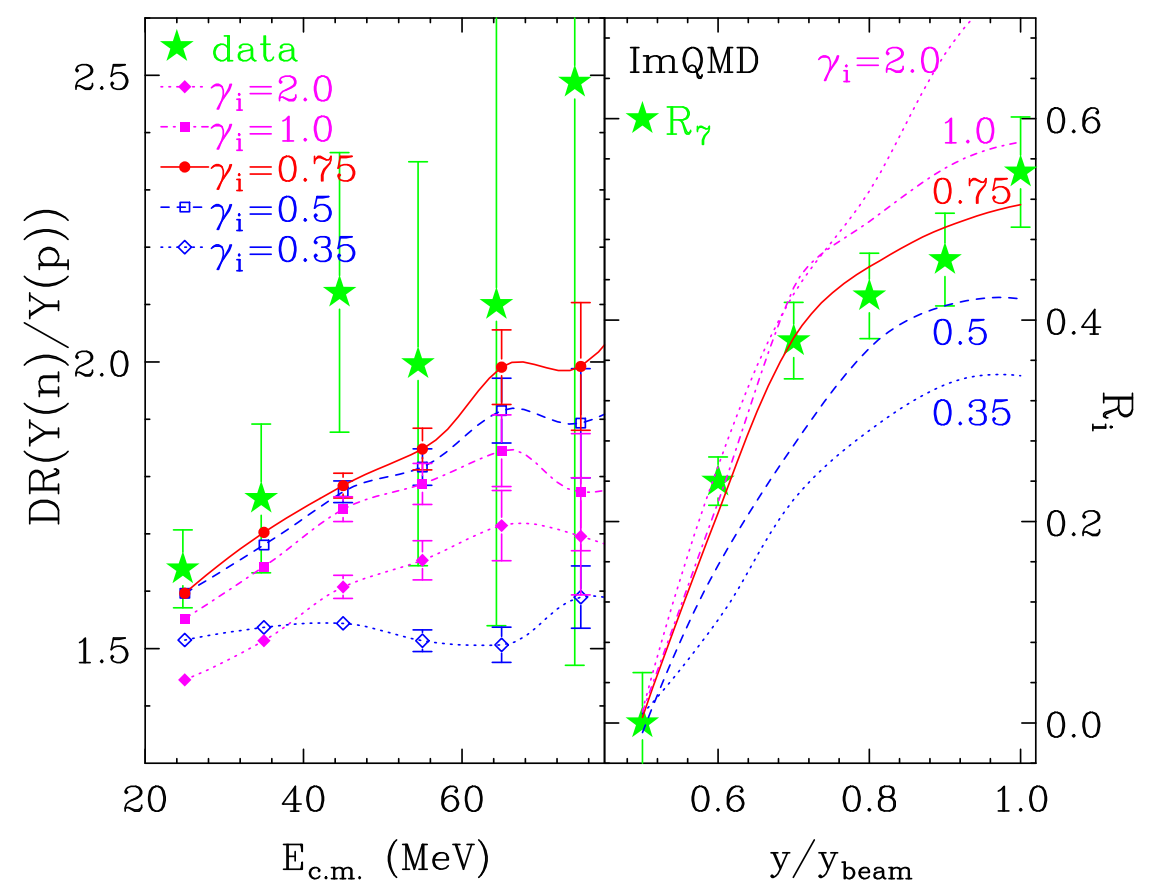

Figure 1: (Color online): Left panel: Comparison of experimental double neutron-proton ratios (star symbols), as a function of nucleon center of mass energy, to ImQMD calculations (lines) with different density dependence of the symmetry energy parameterized in terms of $\gamma_{i}$ in Eq. (1). Right panel: Comparison of experimental isospin transport ratios obtained from the yield of $A=7$ isotopes (star symbols), as a funtion fo the beam rapidity, to ratios from ImQMD calculations (lines) for $\mathrm{b}=6 \mathrm{fm}$.

parameter space. Unfortunately, the CPU requirements for such large-scale ImQMD calculations are too intensive to make such analysis practical. Instead, We have preformed a series of ImQMD calculations at $\mathrm{b}=6 \mathrm{fm}$ with different values of $\gamma_{i}$ over selected values of $S_{0}$ between 25 to $40 \mathrm{MeV}$ to locate the approximate boundaries in the $S_{0}$ and $\gamma_{i}$ plane that satisfy the $2 \sigma$ criterion in the $\chi^{2}$ analysis of the isospin diffusion data. The shaded area shown in the inset of Figure 3 is the result of such analysis.

\section{Comparison of experimental constraints at sub-saturation density}

Near saturation density, one may expand the symmetry energy, $S(\rho)$, about the saturation density, $\rho_{0}$,

$$
S(\rho)=S_{0}+\frac{L}{3} \frac{\rho-\rho_{0}}{\rho_{0}}+\frac{K_{\text {sym }}}{18}\left(\frac{\rho-\rho_{0}}{\rho_{0}}\right)^{2}+\cdots
$$

where $\mathrm{L}$ and $K_{\text {sym }}$ are slope and curvature parameters at $\rho_{0}$. The slope parameter, $\mathrm{L}$, is related to $p_{0}$, the pressure from the symmetry energy for pure neutron matter at saturation density:

$$
L=3 \rho_{0}|d S(\rho) / d \rho|_{\rho_{0}}=\left[3 / \rho_{0}\right] \cdot p_{0}
$$

$p_{0}$, provides the baryonic contribution to the pressure in neutron stars [10], where the energy of symmetric matter, $E_{0}(\rho, \delta=0)$, does not contribute to the pressure. It is also related to the neutron skin thickness $\left(\delta R_{n p}\right)$ of neutron-rich heavy nuclei including ${ }^{208} \mathrm{~Pb}[11,12]$. 


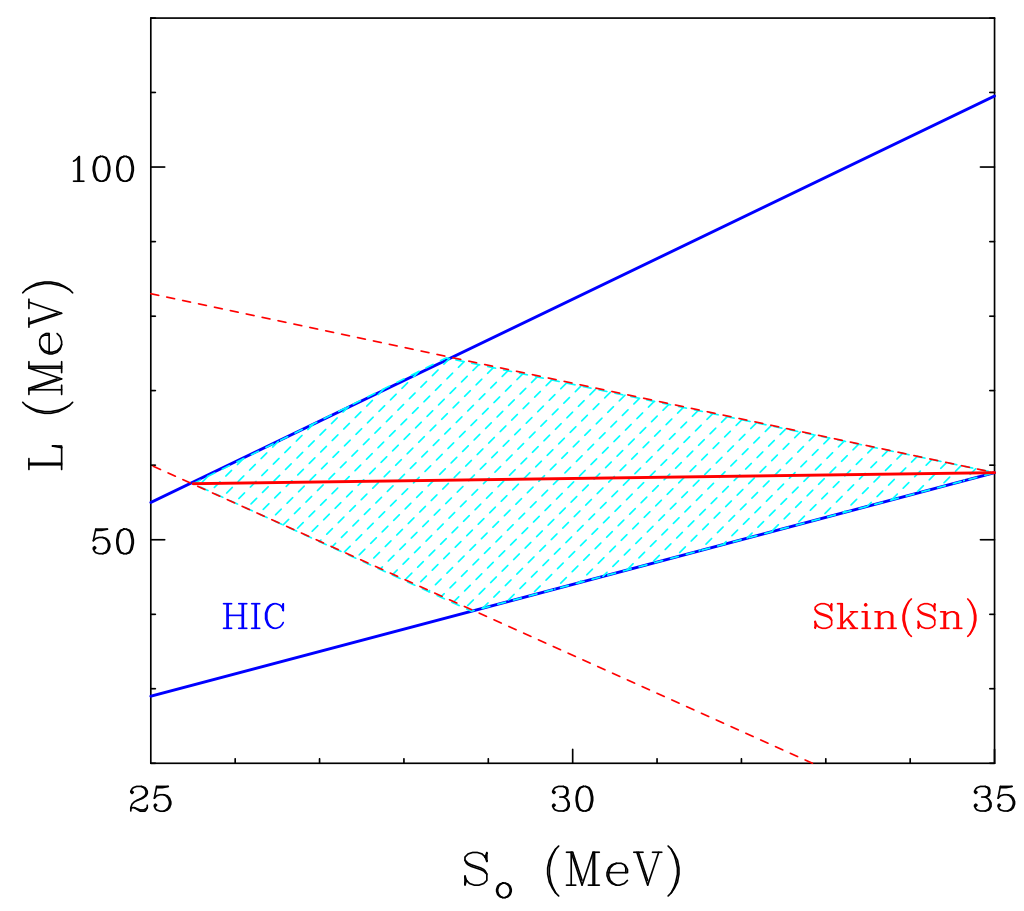

Figure 2: (Color online): Representation of the constraints on the density dependence of symmetry energy as a function of the symmetry energy at saturation density in neutron matter. The hatched areas are extracted from recent analysis of nuclear collisions of Sn nuclei. The two dashed lines represent the constraint boundaries obtained from the analysis of the skin thickness of tin isotopes [13]. The horizontal line indicates the nearly constant mean $L$ value in the overlap region.

The series of ImQMD results calculated at $\mathrm{b}=6 \mathrm{fm}$ with different values of $\gamma_{i}$ over selected values of $S_{0}$ between 25 to $40 \mathrm{MeV}$ give the approximate boundaries in the $S_{0}$ and $L$ plane that satisfy the $2 \sigma$ criterion in the $\chi^{2}$ analysis of the isospin diffusion data. The area bounded by the two solid diagonal lines in Figure 2 and the shaded region in Figure 3 represents a conservative estimate in such effort. Another independent constraint will be needed to provide constraints in $S_{0}$ or $L$.

Recent analysis of the skin thickness of tin isotopes [13] suggests that L decreases with increasing $S_{0}$. The two diagonal dashed lines plotted in Figure 2 represent a $2 \sigma$ analysis of the $\chi^{2}$ discussed in Ref. [13]. Since the trend is opposite to that of the constraints provided by the heavy ion reactions (solid lines), the overlap of the two constraints provide a range of $S_{0}$ and $L$ values. As pointed out by Chen et al, the interesting thing to note is that the mean $L$ values, represented by the horizontal line in Figure 2 obtained from the overlap region of the two constraints, are nearly constant. The range of $L$ and $S_{0}$ values in the overlap region corresponds to a mean skin thickness of ${ }^{208} \mathrm{~Pb}$ of about $0.18 \pm 0.04$ $\mathrm{fm}$ [10]. The range of experimental neutron skin thicknesses of ${ }^{208} \mathrm{~Pb}$ varies from about 0.13 to $0.22 \mathrm{fm}$ [14, 15, 16, 17, 18] which are consistent with results predicted by the constraints.

We have included, in Figure 3, other constraints from the properties of individual nuclei. The corresponding values of pure neutron matter pressure, $p_{0}$, are given in the right axis. The lower box centered at $S_{0}=32.5 \mathrm{MeV}$ depicts the range of $S_{0}$ and $\rho_{0}$ values from analysis of low-lying electric dipole strength (Pygmy Dipole Resonance, PDR) in neutron-rich nuclei ${ }^{68} N i$ and ${ }^{132} S n$ [19, 20]. The upper box centered at $S_{0}=32.5 \mathrm{MeV}$ depicts the constraints reported in Ref. [21] from the analysis of the excitation energies of Isobaric Analog states (IAS). All these constraints overlap significantly with the hatched regions obtained from the heavy ion collisions of tin isotopes as described in section 3 . The 


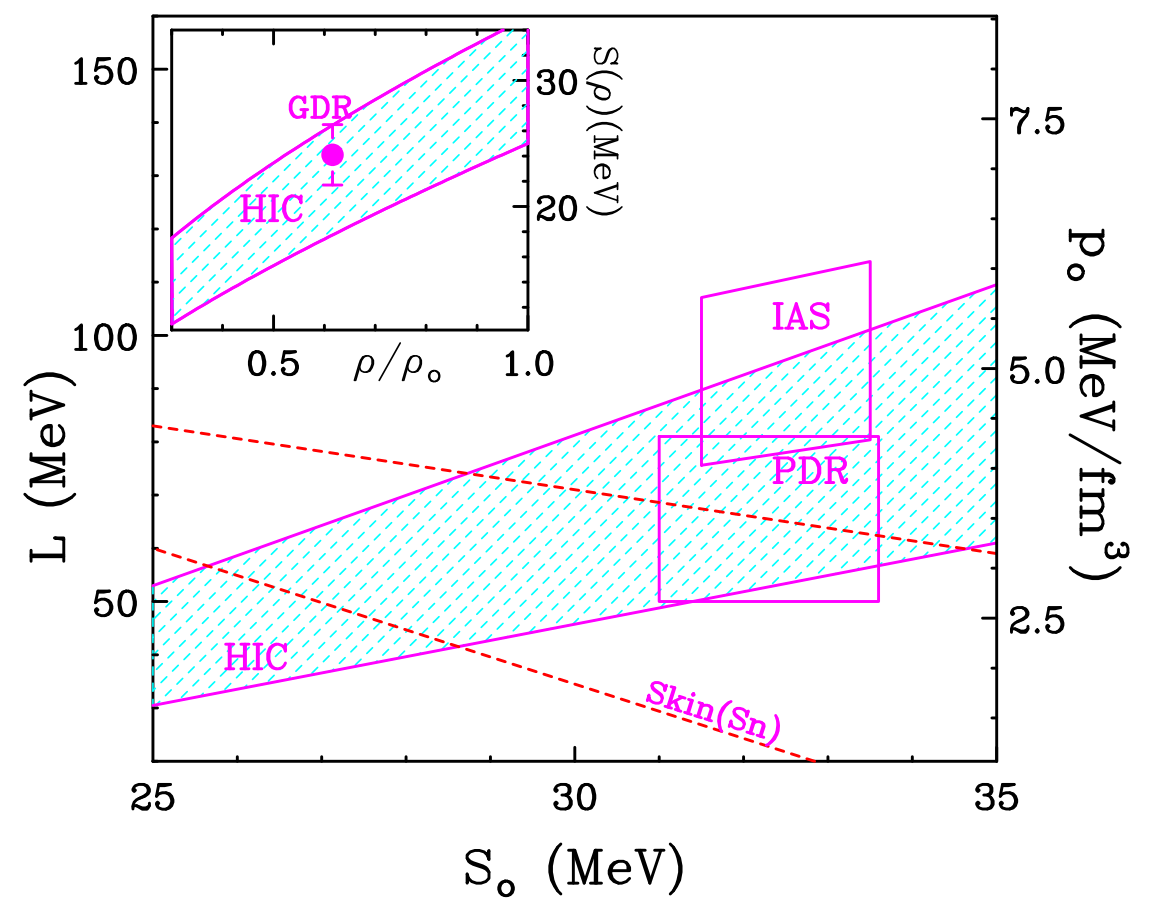

Figure 3: (Color online): Comparison of various constraints on the density dependence of symmetry energy (inset) and pressure as a function of the symmetry energy at saturation density in neutron matter. The hatched areas are extracted from recent analyses of nuclear collisions of Sn nuclei. Open rectangles and the symbol in the inset were obtained from the properties of individual nuclei. The inset shows the density dependence of the symmetry energy of the shaded region. The symbol in the inset represents the GDR results [22].

inset shows what this analysis implies about the density dependence of the symmetry energy. A value for the symmetry energy at around 0.6 normal nuclear matter density, extracted from Giant Dipole Resonance (GDR) data [22], is indicated by the symbol in the inset.

\section{$5 \quad$ Summary and outlook}

In summary, the ability of QMD models to reproduce both isospin diffusion data and double ratio of neutron and proton spectra data is an important step forward in obtaining information about the symmetry energy in heavy-ion collisions. The heavy ion as well as nuclear structure observables examined here provide consistent constraints on the density dependence of the symmetry energy.

To explore the density dependence of the symmetry energy, an international collaboration, consisting of an interdisciplinary, experimental and theoretical team of scientists, has been formed [23] to conduct a series of experiments at unique facilities based in the United States (the National Superconducting Cyclotron Laboratory at Michigan State University), Japan (the Radioactive Ion Beam Factory at RIKEN) and GSI, Germany. Each facility enables the exploration of a different density range. With experiments underway at GSI, better constraints at supra normal density is expected in the near future.

\section{Acknowledgements}

This work has been supported by the U.S. National Science Foundation under Grants PHY-0606007, 0800026, and the Chinese National Science Foundation of China under Grants 10675172. 


\section{References}

[1] P. Danielewicz et al.,Science 298 (2002) 1592

[2] C. Fuchs, Prog. Part. Nucl. Phys. 56 (2006) 1

[3] M. B. Tsang et al., Phys. Rev. Lett. 92 (2004) 062701

[4] T. X. Liu et al., Phys. Rev. C 76 (2007) 034603

[5] M. A. Famiano et al., Phys. Rev. Lett. 97 (2006) 052701.

[6] J. Aichelin et al., Phys. Rev. Lett. 58 (1987) 1926.

[7] Y. Zhang and Z. Li, Phys. Rev. C 74 (2006) 014602.

[8] Y. Zhang, Z. Li and P. Danielewicz, Phys. Rev. C 75 (2007) 034615.

[9] B. A. Li et al., Phys. Rev. Lett. 78 (1997) 1644.

[10] A. W. Steiner, Phys. Rep. 411 (2005) 325

[11] C. J. Horowitz et al., Phys. Rev. Lett. 86 (2001) 5647.

[12] S. Typel and B. A. Brown, Phys. Rev. C 64 (2001) 027302.

[13] L.W. Chen et al., Phys. Rev. C 82 (2010) 024321

[14] A. Krasznahorkay et al., Nucl. Phys. A 567 (1994) 521.

[15] L. Ray, Phys. Rev. C 19 (1979) 1855.

[16] S. Karataglidis et al., Phys. Rev. C 65 (2002) 044306 .

[17] B. Klos et al., Phys. Rev. C 76 (2007) 014311.

[18] V. E. Starodubsky and N. M. Hintz, Phys. Rev. C 49 (1994) 2118.

[19] A. Klimkiewicz et al., Phys. Rev. C 76 (2007) 051603

[20] Andrea Carbone et. al, Phys. Rev. C 81 (2010) 041301(R) .

[21] P. Danielewicz, Jenny Lee Nucl. Phys. A 818 (2009) 36

[22] Luca Trippa et al., Phys. Rev. C 77 (2008) 061304(R) .

[23] http://groups.nscl.msu.edu/hira/sep.htm. 